\title{
AVALIAÇÃO DO DESEMPENHO COGNITIVO EM IDOSOS RESIDENTES EM ZONA RURAL
}

\author{
Juliana Bessa Martins ${ }^{1}$, Celmira Lange $e^{2}$, Marcos Aurélio Matos Lemões ${ }^{3}$, Patrícia Mirapalheta Pereira de \\ Llano $^{4}$, Fernanda dos Santos 5 , Janaína Amorim de Avila ${ }^{5}$
}

\begin{abstract}
RESUMO: O objetivo deste estudo foi descrever o desempenho cognitivo dos idosos residentes na zona rural. Trata-se de uma pesquisa quantitativa descritiva de corte transversal, com idosos de 60 anos ou mais residentes na zona rural de um município do extremo sul do Brasil e ocorreu entre os meses de julho e outubro de 2014. A amostra do estudo foi de 806 idosos. Os participantes da pesquisa foram 450 (55,83\%) do sexo feminino, idosos jovens, com idades entre 60 a 69 anos e possuíam 4 a 7 anos de escolaridade. A média no Mini-Exame do Estado Mental foi de 23,59, desvio padrão 5,21. Homens tiveram média nesta escala maior que mulheres $(p<0,001)$. As diferenças nas médias em relação à idade não foram significativas $(p=0,8)$. Estes resultados poderão servir de subsídios para os profissionais e gestores da área da saúde criem estratégias de prevenção ao declínio cognitivo. DESCRITORES: Envelhecimento; Saúde do idoso; Cognição; Enfermagem. Atenção Primária à Saúde.
\end{abstract}

\section{EVALUATION OF COGNITIVE PERFORMANCE IN OLDER ADULT RESIDENTS OF A RURAL AREA}

\begin{abstract}
The aim of this study was to describe the cognitive performance of older adults living in a rural area.This was a descriptive, quantitative, cross-sectional study, with older adults, aged 60 or more, living in the rural area of a municipality in the south of Brazil, which took place between July and October 2014. The study sample was 806 older adults. The majority of the older adults participants were aged 60-69 years, with 4-7 years of schooling and 450 (55.83\%) were female.The mean in the Mini-Mental State Examination was 23.59, standard deviation 5.21. The men presented a higher mean in this scale than the women $(p<0.001)$. Differences in the mean for age were not significant $(p=0.8)$. These results may serve to support health professionals and managers in the creation of cognitive decline prevention strategies.
\end{abstract}

DESCRIPTORS: Aging; Older adult health; Cognition; Nursing. Primary healthcare.

\section{EVALUACIÓN DEL DESEMPEÑO COGNITIVO EN ANCIANOS QUE VIVEN EN ÁREA RURAL}

RESUMEN: Estudio cuya finalidad fue describir el desempeño cognitivo de los ancianos que viven en espacio rural. Es una investigación cuantitativa descriptiva de cohorte trasversal, con ancianos de 60 años o más que viven en el espacio rural de un municipio del extremo sur de Brasil. Fue realizada entre los meses de julio y octubre de 2014. La muestra del estudio fue de 806 ancianos y los participantes de la investigación totalizaron 450 (55,83\%) del sexo femenino, ancianos jóvenes, con edades entre 60 y 69 años y escolaridade de 4 a 7 años. La media en el Mini Examen del Estado Mental fue de 23,59, desvío patrón 5,21. La media de los hombres en esta escala fue mayor que de mujeres $(p<0,001)$. Las diferencias en las medias referentes a la edad no fueron significativas $(p=0,8)$. Los resultados obtenidos podrán ser utilizados como subsidios para que los profesionales y gestores del área de salud puedan crear estrategias de prevención al declinio cognitivo.

DESCRIPTORES: Envejecimiento; Salud del anciano; Cognición; Enfermería; Atención Primaria a la Salud.

Enfermeira. Residente em Atenção ao Paciente Crítico pela Residência Integrada em Saúde do Grupo Hospitalar Conceição. Porto Alegre, RS, Brasil.

Enfermeira. Doutora em Enfermagem. Docente de Enfermagem e do Programa de Pós-graduação em Enfermagem da Universidade Federal de Pelotas. Pelotas, RS, Brasil.

${ }^{3}$ Enfermeiro. Doutorando em Enfermagem. Universidade Federal de Pelotas. Pelotas, RS, Brasil.

${ }^{4}$ Enfermeira. Doutora em Enfermagem. Universidade Federal de Pelotas. Pelotas, RS, Brasil.

${ }^{5}$ Enfermeira. Doutoranda em Enfermagem. Universidade Federal de Pelotas. Pelotas, RS, Brasil.

Autor Correspondente:

Juliana Bessa Martins

Universidade Federal de Pelotas

Av. Ely Correa, 1328 - 94180-452 - Gravataí, RS, Brasil

Email: julianaabessa@gmail.com
Recebido: $14 / 04 / 2016$

Finalizado: $26 / 07 / 2016$ 


\section{INTRODUÇÃO}

O envelhecimento caracteriza-se por alterações orgânicas que podem resultar em complicações à saúde, comprometer a capacidade cognitiva, e predispor ao aparecimento de doenças neurodegenerativas ${ }^{(1-2)}$.

O déficit cognitivo pode manifestar-se durante o processo de envelhecimento e relacionar-se com as próprias perdas biológicas inerentes ao tempo, à cultura do indivíduo, local de moradia, escolaridade e renda ${ }^{(3)}$. Desta forma, é fundamental que se mantenha adequada cognição, pois permite ao indivíduo compreender e resolver os problemas do cotidiano e nos define como ser pensante ${ }^{(4)}$.

Os déficits cognitivos observados no envelhecimento são os esquecimentos de fatos recentes, dificuldade de efetuar cálculos, mudanças no estado de atenção, diminuição da concentração e do raciocínio, além da lentificação de atividades motoras com redução de habilidades motoras finas ${ }^{(5)}$. $\mathrm{O}$ Mini-Exame do Estado Mental (MEEM) é a escala mais utilizada para o rastreamento do comprometimento cognitivo. Clinicamente, o MEEM tem sido usado para detecção e acompanhamento da evolução de alterações cognitivas, bem como para o monitoramento de eficácia e de efetividade de tratamentos para as mesmas ${ }^{(6)}$.

A população que vive nas zonas rurais está envelhecendo à semelhança dos que vivem nas zonas urbanas, porém, estudos com esta população apontam para uma realidade onde predomina a pobreza, isolamento, baixos níveis educacionais, residências mais precárias, limitações de transporte, problemas crônicos de saúde e distância dos recursos sociais e de saúde ${ }^{(7)}$.

O município de Pelotas, no estado do Rio Grande do Sul, tem 328.275 habitantes, aproximadamente 22 mil pessoas vivem na zona rural, e destas 15,8\% têm mais de 60 anos $^{(8)}$. Esta população deve ter especial atenção, pois as doenças que apresentam podem ser diferentes, em função das condições do ambiente, da rede de serviços de saúde disponíveis e das características socioeconômicas ${ }^{(9)}$. A população rural muitas vezes é prejudicada em relação ao acesso dos serviços de saúde pela escassez nestas localidades, sendo necessário maior deslocamento, diferente da zona urbana em que geralmente os serviços estão próximos. Diante deste fato, autores afirmam que devido a esta dificuldade de acesso, os idosos rurais podem ter a qualidade de vida comprometida, na qual está incluída a avaliação do desempenho cognitivo ${ }^{(10)}$.

Diante do exposto, percebe-se a necessidade da realização de estudos epidemiológicos de rastreamento do declínio cognitivo entre a população idosa rural, pois estes são dispositivos importantes e podem se constituir em um instrumento relevante para potencializar o preconizado nas políticas públicas de atenção à saúde para o idoso.

Portanto, as singularidades dos idosos residentes no meio rural, foco deste trabalho, apontam a necessidade de desenvolver pesquisas e ações que promovam a sua capacidade de adaptação nesse contexto, com o intuito de minimizar as incapacidades auferidas em decorrência do envelhecimento ${ }^{(11)}$.

Considerando os aspectos supracitados e entendendo a importância para as políticas públicas ao idoso, e a organização da rede de serviços de saúde de avaliar o estado cognitivo durante o envelhecimento, o presente estudo tem como objetivo descrever o desempenho cognitivo dos idosos residentes na zona rural.

\section{- MÉTODO}

Trata-se de uma pesquisa quantitativa descritiva de corte transversal com idosos de 60 anos ou mais. Participaram 806 idosos residentes na zona rural do município de Pelotas, estado do Rio Grande do Sul (RS), cadastrados em dez Unidades Básicas de Saúde (UBS) que possuem Estratégia de Saúde da Família (ESF).

Os critérios de inclusão foram possuir 60 anos ou mais, residir na zona rural de Pelotas, ter sido contemplado no sorteio realizado pelas pesquisadoras e aceitar divulgação dos dados. Foram excluídos 
os indivíduos que, no momento da entrevista para pesquisa, estivessem viajando, privados de liberdade por decisão judicial, residindo em Instituições de Longa Permanência e hospitalizados.

Após contato com as UBS, por meio do enfermeiro responsável, realizou-se uma lista com os nomes e números dos prontuários dos idosos. Em seguida, foi realizado um cálculo proporcional distribuído entre as dez UBS, gerando a quantidade necessária de idosos para entrevistas. O cálculo do tamanho da amostra foi de 468 idosos, com nível de significância de 5\%, e poder estatístico de 95\%. No entanto, foram entrevistados 806 idosos, ou seja, o poder estatístico neste estudo foi superior a 95\%.

O instrumento da pesquisa apresentava questões fechadas e pré-codificadas de dados demográficos e socioeconômicos, além da aplicação da escala do MEEM. A entrevista foi realizada na residência dos idosos, para minimizar perdas por dificuldades em locomoção do idoso. Para este estudo, foram utilizados os escores do Ministério da Saúde do Brasil (2007) que considera os pontos de corte para: analfabetos com pontuação igual a 19, de um a três anos de escolaridade igual a 23, de quatro a sete anos de escolaridade igual a 24 e maior que sete anos de escolaridade igual a 28 pontos $^{(12)}$.

O MEEM é um instrumento de rastreio, contendo questões agrupadas em sete categorias que avaliam os seguintes domínios: orientação temporal (5 pontos), orientação espacial (5 pontos), memória imediata e de evocação (3 pontos), atenção e cálculo (5 pontos), recordação das três palavras ( 3 pontos), linguagem (8 pontos) e capacidade construtiva visual (1 ponto). O escore do MEEM pode variar de um mínimo de zero ponto, o qual indica o maior grau de comprometimento cognitivo dos indivíduos, até um total máximo de 30 pontos, que por sua vez, corresponde a melhor capacidade cognitiva. O MEEM não serve para diagnóstico, mas serve para indicar que funções devem ser melhor investigadas. É um dos poucos testes validados e adaptados para a população brasileira ${ }^{(12)}$.

A escala do MEEM sendo uma avaliação cognitiva foi respondida exclusivamente pelo próprio idoso, enquanto as outras questões poderiam ser respondidas ou auxiliadas por um respondente auxiliar ou substituto.

As variáveis independentes foram: sexo (feminino/masculino), idade (anos completos), se frequentou a escola (sim/não), anos de estudo (1-3 anos, 4-7 anos e mais de 7 anos). Os respondentes auxiliares colaboraram na pesquisa quando o idoso não compreendia a pergunta, geralmente por déficit auditivo ou não saber precisar dados econômicos, medicamentos, entre outras. E os respondentes substitutos respondiam as questões caso o idoso apresentasse déficit cognitivo, limitações físico-funcionais ou não falasse português, pois se tratava de uma zona de colonização de alemães, italianos, pomeranos e outras etnias.

A pesquisa desenvolveu-se entre os meses de julho e outubro de 2014. Para assegurar a qualidade das informações, foi realizada a revisão das codificações do questionário e a dupla digitação simultânea no banco de dados.

Os dados coletados foram digitados no Software Epi Info versão 6.04 ${ }^{\circledast}$, sob forma de dupla entrada. Para verificação da consistência interna e análise foram realizadas no programa STATA $11.1^{\circledR}$. A análise dos resultados foi descritiva. Houve utilização do Teste ANOVA para análise de variância e do teste de hipóteses Teste t de student. Foi avaliado o desempenho cognitivo dos idosos residentes na zona rural, segundo dados de sexo, faixa etária e escolaridade com a média de resultados do MEEM.

O presente estudo foi aprovado pelo Comitê de Ética em Pesquisa sob protocolo número 649.802/2014, seguindo todos os preceitos da Resolução do Conselho Nacional de Saúde nº 466/2012(13), e conduzido de acordo com os padrões éticos exigidos.

\section{RESULTADOS}

Analisando as características da população residente da zona rural do município de Pelotas/RS que participaram da pesquisa (Tabela 1), observou-se que 450 (55,83\%) idosos pertenciam ao sexo feminino de um total de 806 entrevistados. Ao analisar a faixa etária, a idade dos idosos variou de 60 a 99 anos, 444 (54,09\%) possuíam idades entre 60 a 69 anos. A média de idade desta amostra foi 70,66 anos e DesvioPadrão (DP) de 7,51. 
Em relação à escolaridade, $379(47,02 \%)$ idosos possuíam quatro a sete anos de escolaridade. A média de escolaridade dos entrevistados foi 3,57 anos (DP=2,62).

Tabela 1 - Características da amostra de idosos moradores da zona rural, de acordo com sexo, faixa etária e escolaridade. Pelotas, RS, Brasil, $2014(\mathrm{~N}=806)$

\begin{tabular}{lc} 
Variáveis & $\mathbf{N}(\%)$ \\
\hline Sexo & \\
\hline Masculino & $356(44,17)$ \\
\hline Feminino & $450(55,83)$ \\
\hline Faixa etária (anos) & \\
\hline 60 a 69 & $444(54,09)$ \\
\hline 70 a 79 & $264(32,75)$ \\
\hline 80 a 89 & $88(10,92)$ \\
\hline 90 ou mais & $10(1,24)$ \\
\hline Escolaridade & \\
\hline Analfabeto & $111(13,77)$ \\
\hline 1 a 3 anos & $270(33,50)$ \\
\hline 4 a 7 anos & $379(47,02)$ \\
\hline Mais que 7 anos & $46(5,71)$ \\
\hline Total & $\mathbf{8 0 6}(\mathbf{1 0 0})$
\end{tabular}

Nesta amostra, obteve-se média do Mini-Exame do Estado Mental de 23,59 (DP=5,21), sendo que a pontuação pode variar de zero a 30 pontos.

Ao analisar a proporção dos idosos da zona rural, com e sem déficit cognitivo de acordo com a escolaridade e os pontos de corte do MEEM (Tabela 2), 28 (60,87\%) idosos com escolaridade de sete anos ou mais apresentaram déficit. O maior percentual de déficit cognitivo, analisando a proporção, esteve presente nos idosos com mais anos de estudo.

Tabela 2 - Distribuição dos idosos da zona rural, com e sem déficit cognitivo, segundo escolaridade. Pelotas, RS, Brasil, $2014(\mathrm{~N}=806)$

\begin{tabular}{llcccc} 
Variável & Classificação & \multicolumn{4}{c}{ Escolaridade } \\
\cline { 3 - 6 } & & $\begin{array}{c}\text { Analfabeto } \\
\mathbf{N}(\%)\end{array}$ & $\begin{array}{c}\mathbf{1} \text { a } \mathbf{3} \text { anos } \\
\mathbf{N}(\%)\end{array}$ & $\begin{array}{c}\mathbf{4} \text { a } \mathbf{7} \text { anos } \\
\mathbf{N}(\%)\end{array}$ & $\begin{array}{c}\text { Mais que } \mathbf{7} \text { anos } \\
\mathbf{N}(\%)\end{array}$ \\
\hline Déficit Cognitivo & Não & $60(54,05)$ & $140(51,85)$ & $249(65,70)$ & $18(39,13)$ \\
\cline { 2 - 6 } & Sim & $51(45,95)$ & $130(48,15)$ & $130(34,30)$ & $28(60,87)$ \\
\hline Total & & $\mathbf{1 1 1}$ & $\mathbf{2 7 0}$ & $\mathbf{3 7 9}$ & $\mathbf{4 6}$
\end{tabular}

A Tabela 3 descreve a distribuição das médias e desvios-padrões do MEEM de acordo com o sexo, faixa etária e escolaridade.

De acordo com o sexo, os idosos homens tiveram média do MEEM $(24,89, \mathrm{DP}=4,70)$ maior que das mulheres $(22,56, D P=5,37)$, apresentando significância estatística $(p<0,001)$. No que se refere à faixa etária, as diferenças nas médias do MEEM em relação à idade não foi significativa $(p=0,8)$. A menor média do MEEM para os idosos de 80 a 89 anos foi 23,42 (DP=5,99).

O escore do MEEM apresentou média diretamente proporcional à escolaridade. Os idosos analfabetos obtiveram média do MEEM de 19,75 ( $\mathrm{DP}=5,05)$, com significância estatística $(\mathrm{p}<0,001)$. 
Tabela 3 - Distribuição das médias e desvios-padrão do Mini-Exame do Estado Mental (MEEM) de acordo com sexo, faixa etária e escolaridade. Pelotas, RS, Brasil, $2014(\mathrm{~N}=806)$

\begin{tabular}{lcccc} 
Variáveis & Média MEEM & Desvio Padrão & N & Valor de $\mathbf{p}$ \\
\hline Sexo & & & & \\
\hline Masculino & 24,89 & 4,7 & 356 & $\mathrm{p}<0,001^{*}$ \\
\hline Feminino & 23,56 & 5,37 & 450 & \\
\hline Faixa etária (anos) & & & & \\
\hline 60 a 69 & 23,65 & 5 & 444 & $\mathrm{p}=0,8^{* *}$ \\
\hline 70 a 79 & 23,49 & 5,36 & 264 & \\
\hline 80 a 89 & 23,42 & 5,99 & 88 & \\
\hline 90 ou mais & 25,1 & 3,07 & 10 & \\
\hline Escolaridade & & & & \\
\hline Analfabeto & 19,75 & 5,05 & 111 & $\mathrm{p}<0,001^{* *}$ \\
\hline 1 a 3 anos & 22,79 & 4,81 & 270 & \\
\hline 4 a 7 anos & 24,77 & 5,03 & 379 & \\
\hline Mais que 7 anos & 27,86 & 1,75 & 46 &
\end{tabular}

* Teste T Pareado

** Teste ANOVA

\section{DISCUSSÃO}

Em relação aos dados sociodemográficos, houve predominância do gênero feminino, caracterizando a feminização do envelhecimento ${ }^{(14)}$. Ao apontar que existem mais mulheres que homens na amostra podemos considerar as características de colonização deste estudo predominantemente de origem alemã, fato que pode influenciar no estilo de vida familiar, incidindo na fixação de mulheres na área rural $^{(15)}$. Provavelmente esta predominância tenha ocorrido neste estudo, uma vez que as mulheres vivem com seus companheiros e as viúvas vivem com os filhos, permanecendo assim na zona rural, local onde fixam as relações de amizade. Possivelmente, esta realidade seja diferente hoje, e as mulheres busquem novas oportunidades na cidade.

Outros estudos contrariam os dados obtidos nesta pesquisa, no qual identificam que os homens tendem a permanecer mais nas áreas rurais do que as mulheres, podendo ser evidenciada pelos processos migratórios brasileiros, pois as mulheres saem do campo em direção aos centros urbanos buscando atividades menos árduas, além de mudanças residenciais, buscando a proximidade dos filhos, moradores de áreas urbanas ${ }^{(11,16)}$.

Com referência ao sexo, resultados sugerem que características particulares de cada população podem ter influência na associação entre função cognitiva e $\operatorname{sexo}^{(9)}$. As análises do MEEM com variáveis sociodemográficas confirmam com resultados de outros estudos, como no estudo multicêntrico realizado nos municípios de Ribeirão Preto, estado de São Paulo e Caxias do Sul, no estado do Rio Grande do Sul ${ }^{(17)}$, nos quais idosos do sexo masculino obtiveram melhor desempenho no MEEM. Este estudo apresentou perdas cognitivas entre as idosas, que tiveram pontuação mais baixa no MEEM, visto que as mulheres tiveram mais dificuldades na realização de cálculos, atividade realizada facilmente pelos homens, que incluía frequentar bancos, realizar cálculos e pagamentos. Portanto, entende-se a necessidade de orientar os profissionais de saúde para atentar as questões de gênero ao atender a população idosa.

A média etária deste estudo foi concordante ao estudo realizado com dados demográficos e escores do MEEM de 968 indivíduos ${ }^{(18)}$, os quais possuíam idades variando de 60 a 92 anos entre os idosos, possuindo média de idade da amostra total de 70,6 (DP=7,3) anos. Este dado se aproxima das estimativas oficiais de expectativa de vida no Brasil, que é de $74,9 \operatorname{anos}^{(19)}$.

De acordo com a faixa etária, as diferenças nas médias do MEEM em relação à idade não foram significativas neste estudo $(p=0,8)$, contrariando outros autores que apontam que a idade é um dos 
determinantes mais importantes do declínio cognitivo. Estudo de base populacional tem mostrado de forma consistente que existe uma piora do desempenho no MEEM com o aumento da idade ${ }^{(6)}$. Estudo em Dourados, Mato Grosso do Sul ${ }^{(14)}$, em unidades na zona urbana e zona rural, mostrou que a prevalência de déficit cognitivo foi maior para os idosos na faixa etária acima de 80 anos $(68,4 \%)$ do que nos idosos na faixa etária de 70 a 79 anos $(41,4 \%)$ e os da faixa de 60 a 69 anos $(35,2 \%),(p<0,001)$. Assim como estes autores, outros também averiguaram que a prevalência de déficit cognitivo aumenta conforme o avançar da idade ${ }^{(20)}$.

Nesta pesquisa, a maioria dos idosos possuía de quatro a sete anos de escolaridade, com média de 3,57 anos, porém, de acordo com a contextualização histórica da população de idosos de origem alemã, supõe-se que, apesar das dificuldades impostas pelo Estado, os idosos que iniciaram seus estudos na década de 50 tinham maior acesso à formação básica. Isto se deu, possivelmente, com criação das escolas comunitárias que disponibilizavam a comunidade a formação no ensino básico, o que justifica a razão pela qual os idosos da zona rural de Pelotas apresentarem um nível de escolaridade alto para os padrões da época.

Outro estudo realizado com idosos em uma comunidade rural apresentou dados semelhantes: de 39 idosos entrevistados, 55,9\% $(n=19)$ dos respondentes possuíam até três anos de estudo e a proporção de mulheres com escolaridade entre um e sete anos $(89,5 \%, n=17)$ foi superior à de homens $(73,4 \%, n=11)^{(11)}$. Este é um fato esperado para a população idosa, pois há algumas décadas no Brasil, no que se refere à área rural, eram poucas as possibilidades de estudar e havia dificuldade no acesso à educação ${ }^{(21)}$.

A média de escolaridade encontrada nesta pesquisa, de 3,57 anos de estudo, corrobora com resultados de outros estudos rurais, em que a maior parte dos participantes possuía até quatro anos de estudo ${ }^{(16,22)}$ e refletindo dificuldades no acesso à educação na área rural.

A média do escore do MEEM encontrada neste estudo 23,59 $(\mathrm{DP}=5,21)$ foi próxima à média da amostra de um estudo realizado na Austrália, cuja pontuação média foi de $21,1(\mathrm{DP}=6,1)^{(23)}$. E de um estudo realizado em uma comunidade rural do Rio Grande do Sul, que obteve média geral de 25,8 $(\mathrm{DP}= \pm 2,8)\left({ }^{10)}\right.$.

Nesta pesquisa o maior percentual de déficit cognitivo se manteve nos idosos com mais anos de estudo. Diferentemente do estudo realizado com 350 idosos, residentes em áreas de cobertura das equipes urbanas da ESF, que apontou maior desenvolvimento de déficit cognitivo no grupo de idosos sem escolaridade. Ainda, mostrou que entre os 76 idosos sem escolaridade, encontraram-se quatro (5,3\%) com déficit cognitivo e entre os de escolaridade superior não se evidenciaram pessoas portadoras de declínio cognitivo ${ }^{(24)}$.

Supõe-se, portanto, que o ponto de corte muito rígido para aqueles com mais de sete anos de estudo segundo (MS) pode legitimar o desfecho de tantos idosos com déficit cognitivo nesta faixa de escolaridade. Amparado que, na zona rural, a rotina de trabalho é sempre igual (independente de sábado, domingo e feriado) e a luz do dia guia as atividades. Portanto, é possível que houvesse um viés de recordatório sobre dia da semana, mês e/ou hora, ao errar estas questões, a pontuação será 27 no MEEM e os idosos com mais de 7 anos de escolaridade já serão considerados como tendo déficit cognitivo.

O escore do MEEM apresentou média diretamente proporcional à escolaridade neste estudo, de forma significativa $(<0,001)$, porém, ao analisar o MEEM de acordo com a escolaridade e os pontos de corte do Ministério da Saúde, os idosos com maior número de anos de escolaridade apresentaram maior percentual de déficit cognitivo. Concordante com um estudo de caráter transversal, realizado com 120 idosos $^{(3)}$, no qual a baixa escolaridade influenciou de forma negativa o desempenho no MEEM. A pontuação média no MEEM dos idosos mais escolarizados foi significativamente maior em comparação àqueles que não frequentaram a escola (24,86 versus 19,17; $p<0,001)$.

Em outro estudo ${ }^{(25)}$, os indivíduos sem escolaridade formal tiveram performance significativamente pior que os com um a quatro anos de escolaridade $(p<0,0001)$ e os com mais de cinco anos de escolaridade $(p<0,0001)$. Entretanto, não foi observada diferença entre estes dois últimos grupos de escolaridade $(p=0,248)$. 
Para outros autores ${ }^{(26)}$, a idade e a educação foram significativamente associados com o desempenho no teste MEEM. Em diferentes subgrupos de idade e educação, mudanças de pelo menos dois a três pontos indicam variações significativas de testes do MEEM no nível de confiança de $90 \%$.

Percebe-se que a escolaridade é um fator muito importante para a determinação dos escores finais no MEEM. No Brasil, o ensino fundamental é bastante heterogêneo, com características regionais próprias, como número de dias na escola, período de horas diárias e frequência de educadores ${ }^{(12)}$. Isto faz com que haja heterogeneidade nas respostas, principalmente nos grupos de escolaridade inferior ${ }^{(14)}$.

Alguns autores afirmam que, quanto maior o nível de escolaridade, mais difícil o desenvolvimento de quadros demenciais ${ }^{(5-6,27)}$. A escolaridade exerce papel importante sobre o desempenho cognitivo, bem como influencia de forma significativa os resultados do MEEM, ou seja, quanto maior o nível de escolaridade, maiores escores se atinge ${ }^{(17)}$. Também é um indicador preciso por estar relacionado às possibilidades de acesso aos serviços de saúde e ao emprego, enquanto que o analfabetismo causa susceptibilidade maior à dependência ${ }^{(6,24)}$.

Portanto, identificar as condições cognitivas de saúde dos idosos possibilitará intervenções direcionais, de forma a atender suas demandas e melhorar sua qualidade de $v^{2}{ }^{(24)}$.

O idoso inserido no contexto rural, além das peculiaridades do processo de envelhecimento, traz consigo uma série de características próprias do meio rural. Assim, os profissionais de saúde que atuam neste contexto devem desenvolver habilidades específicas de atuação, visto as situações às quais são expostos continuamente. O profissional deve utilizar de forma ampliada suas habilidades para assistir de forma integral o idoso, incluindo aspectos culturais no cuidado ${ }^{(28)}$.

Neste sentido, a enfermagem possui papel muito importante, pois é de sua prática profissional organizar e executar atividades individuais e coletivas de promoção da saúde e de prevenção de morbidades, por meio da Estratégia Saúde da Família, modelo adotado na Atenção Básica do Brasil.

\section{CONSIDERAÇÕES FINAIS}

Os idosos da comunidade estudada eram 55,83\% do sexo feminino, idosos jovens (60-69 anos), possuíam quatro a sete anos de escolaridade. A média do MEEM nesta amostra foi diretamente proporcional à escolaridade, porém ao analisar em proporção, considerando a escolaridade e os pontos de corte do MEEM proposto pelo Ministério da Saúde, a maior parte dos idosos com mais de sete anos de estudo apresentou déficit cognitivo.

O escore do MEEM apresentou média diretamente proporcional à escolaridade, apontando que a escolaridade exerce um papel importante sobre o desempenho em tarefas neuropsicológicas e pode influenciar de forma positiva nos resultados do MEEM. No que se refere à faixa etária, as diferenças nas médias do MEEM em relação à idade não foi significativa neste estudo.

Ao analisar o desempenho cognitivo da população de idosos na zona rural, os homens apresentaram média do MEEM maior que a média das mulheres. Este resultado assinala que as características particulares da população da zona rural podem influenciar na associação entre função cognitiva e sexo.

Os resultados possibilitam conhecer as condições de saúde da população idosa rural a respeito de seu desempenho cognitivo e podem servir de subsídios para os profissionais e gestores da área da saúde no desenvolvimento de estratégias de planejamento, prevenção de doenças e agravos buscando proporcionar um envelhecimento ativo e com qualidade de vida para esta população.

Como limitação do estudo destaca-se o delineamento do estudo transversal, o qual não permite fazer inferências causais, pois não dá para distinguir a temporalidade dos fatos de exposição e desfecho, assim como a análise bi-variada limita a realização do controle para fatores de confusão.

Almeja-se que os resultados desta pesquisa possam contribuir para a melhoria do cuidado aos idosos da população rural e estimular outros estudos que incluam a população idosa domiciliada na 
zona rural com o intuito de apontar os agravos e patologias mais recorrentes, para assim planejar e buscar maior eficiência ao atender as demandas das pessoas moradoras nestas áreas.

\section{REFERÊNCIAS}

1. Ferreira LS, Pinho MSP, Pereira MWM, Ferreira AP. Perfil cognitivo de idosos residentes em Instituições de longa Permanência de Brasília-DF. Rev. bras. enferm. [Internet] 2014; 67(2) [acesso em 04 set 2014]. Disponível: http://dx.doi.org/10.5935/0034-7167.20140033.

2. Leite MT, Hildebrandt LM, Kirchner RM, Winck MT, da Silva LAA, Franco GP. Estado cognitivo e condições de saúde de idosos que participam de grupos de convivência. Rev. Gaúcha Enferm. [Internet] 2012; 33(4) [acesso em 04 set 2014]. Disponível: http://dx.doi.org/10.1590/S1983-14472012000400008.

3. de Paula AFM, Ribeiro LHM, D'Elboux MJ, Guariento ME. Avaliação da capacidade funcional, cognição e sintomatologia depressiva em idosos atendidos em ambulatório de Geriatria. Rev Bras Clin Med. [Internet] 2013; 11(3) [acesso em 10 set 2014]. Disponível: http://files.bvs.br/upload/S/1679-1010/2013/v11n3/a3767.pdf.

4. de Moraes EN. Organização Pan-Americana da Saúde. Atenção à saúde do Idoso: Aspectos Conceituais. [Internet] Brasília: Organização Pan-Americana da Saúde; 2012 [acesso em 10 set 2014]. Disponível: http:// apsredes.org/site2012/wp-content/uploads/2012/05/Saude-do-Idoso-WEB1.pdf.

5. Bertolucci PHF, Minett TSC. Perda de memória e demência. In: Prado FC, Ramos J, Valle JR, organizadores. Atualização terapêutica. São Paulo: Artes Médicas; 2007. p. 8.

6. Valle EA, Castro-Costa E, Firmo JOA, Uchoa E, Lima-Costa MF. Estudo de base populacional dos fatores associados ao desempenho no Mini Exame do Estado Mental entre idosos: Projeto Bambuí. Cad. Saúde Pública. [Internet] 2009; 25(4) [acesso em 29 set 2014]. Disponível: http://dx.doi.org/10.1590/S0102-311X2009000400023.

7. de Morais EP, Rodrigues RAP, Gerhardt TE. Os idosos mais velhos no meio rural: realidade de vida e saúde de uma população do interior gaúcho. Texto Contexto Enferm. [Internet] 2008; 17(2) [acesso em 20 jan 2015]. Disponível: http://dx.doi.org/10.1590/S0104-07072008000200021.

8. Brasil. Instituto Brasileiro de Geografia e Estatística (IBGE). Distribuição da população por sexo segundo os grupos de idade Brasil (RS). [Internet] 2010 [acesso em 20 set de 2014]. Disponível: http://www.censo2010.ibge. gov.br/sinopse/index.php? $\mathrm{uf}=43 \&$ dados $=26$.

9. de Morais EP. Envelhecimento no meio rural: condições de vida, saúde e apoio dos idosos mais velhos de Encruzilhada do Sul-RS [tese]. Ribeirão Preto (SP): Universidade de São Paulo; 2007.

10. Tavares DMS, Gomes NC, Dias FA, Santos NMF. Fatores associados à qualidade de vida de idosos com osteoporose residentes na zona rural. Esc. Anna Nery. [Internet] 2012; 16(2) [acesso em 5 fev 2015]. Disponível: http://dx.doi.org/10.1590/S1414-81452012000200023.

11. Rigo II, Paskulin LMG, de Morais EP. Capacidade funcional de idosos de uma comunidade rural do Rio Grande do Sul. Rev. Gaúcha Enferm. [Internet] 2010; 31(2) [acesso em 5 fev 2015]. Disponível: http://dx.doi.org/10.1590/ S1983-14472010000200008.

12. Ministério da Saúde (BR). Secretaria de Atenção à Saúde. Departamento de Atenção Básica. Envelhecimento e saúde da pessoa idosa. [Internet] Brasília: Ministério da Saúde; 2007 [acesso em 26 fev 2016]. Disponível: http:// bvsms.saude.gov.br/bvs/publicacoes/abcad19.pdf.

13. Ministério da Saúde (BR). Conselho Nacional de Saúde. Diretrizes e normas regulamentadoras de pesquisa envolvendo seres humanos. Resolução n. 466, de 12 de dezembro de 2012. Brasília; 2012.

14. Santos CS, Cerchiari EAN, Alvarenga MRM, Faccenda O, Oliveira MAC. Avaliação da confiabilidade do miniexame do estado mental em idosos e associação com variáveis sociodemográficas. Cogitare Enferm. [Internet] 2010; 15(3) [acesso em 26 fev 2016] Disponível: http://dx.doi.org/10.5380/ce.v15i3.18879.

15. Dal Pizzol TS, Pons ES, Hugo FN, Bozzetti MC, de Sousa MLR, Hilgert JB. Uso de medicamentos entre idosos residentes em áreas urbanas e rurais de município no Sul do Brasil: um estudo de base populacional. Cad. Saúde Pública. [Internet] 2012; 28(1) [acesso em 20 fev 2015]. Disponível: http://dx.doi.org/10.1590/S0102- 
$311 \times 2012000100011$.

16. Silva JLA. O idoso do município de Arambaré - RS um contexto rural de envelhecimento [dissertação]. Rio Grande do Sul (RS). Universidade Federal do Rio Grande do Sul; 2005.

17. Rosset I, Roriz-Cruz M, Santos JLF, Haas VJ, Fabrício-Wehbe SCC, Rodrigues RAP. Diferenciais socioeconômicos e de saúde entre duas comunidades de idosos longevos. Rev. Saúde Pública. [Internet] 2011; 45(2) [acesso em 15 jan 2016]. Disponível: http://dx.doi.org/10.1590/S0034-89102011000200018.

18. Kochhann R, Varela JS, Lisboa CSM, Chaves MLF. The Mini Mental State Examination: Review of cutoff points adjusted for schooling in a large Southern Brazilian sample. Dement. Neuropsychol. [Internet] 2010; 4(1) [acesso em 20 out 2014]. Disponível: http://www.demneuropsy.com.br/detalhe_artigo.asp?id=199.

19. Brasil. Instituto brasileiro de geografia e Estatística (IBGE). Tábua Completa de Mortalidade - Ambos os Sexos. [Internet] 2013 [acesso em 20 out 2014]. Disponível: ftp://ftp.ibge.gov.br/Tabuas_Completas_de_Mortalidade/ Tabuas_Completas_de_Mortalidade_2013/pdf/ambos_pdf.pdf.

20. Holz AW, Nunes BP, Thumé E, Lange C, Facchini LA. Prevalência de déficit cognitivo e fatores associados entre idosos de Bagé, Rio Grande do Sul, Brasil. Rev. bras. epidemiol. [Internet] 2013; 16(4) [acesso em 20 nov 2015$].$ Disponível: http://dx.doi.org/10.1590/S1415-790X2013000400008.

21. Valcarenghi RV, Santos SSC, Barlem ELD, Pelzer MT, Gomes GC, Lange C. Alterações na funcionalidade/ cognição e depressão em idosos institucionalizados que sofreram quedas. Acta paul. enferm. [Internet] 2011; 24(6) [acesso em 25 fev 2015). Disponível: http://dx.doi.org/10.1590/S0103-21002011000600017.

22. Alcântara LR. Idosos rurais: fatores que influenciam trajetórias e acesso a serviços de saúde no município de Santana da Boa Vista/RS [dissertação]. Porto Alegre: Escola de Enfermagem. Universidade Federal do Rio Grande do Sul; 2009.

23. Sachdev PS, Levitan C, Crawford J, Sidhu M, Slavin M, Richmond R, et al. The Sydney Centenarian Study: methodology and prole of centenarians and near-centenarians. Int. Psychogeriatr. [Internet] 2013; 25(6) [acesso em 15 jan 2016]. Disponível: http://dx.doi.org/10.1017/S1041610213000197.

24. Faria EC, da Silva SA, de Farias KRA, Cintra A. Avaliação cognitiva de pessoas idosas cadastradas na Estratégia Saúde da Família: município do Sul de Minas. Rev. esc. enferm. USP. [Internet] 2011; 45(n. esp2) [acesso em 20 nov 2015]. Disponível: http://dx.doi.org/10.1590/S0080-62342011000800019.

25. Diniz BSO, Volpe FM, Tavares AR. Nível educacional e idade no desempenho no Miniexame do Estado Mental em idosos residentes na comunidade. Rev. psiquiat. clín. [Internet] 2007; 34(1) [acesso 10 set 2015]. Disponível: http://dx.doi.org/10.1590/S0101-60832007000100002.

26. Stein J, Luppa M, Maier W, Wagner M, Wolfsgruber S, Scherer M, et al. Assessing cognitive changes in the elderly: Reliable Change Indices for the Mini-Mental State Examination. Acta Psychiatr Scand. [Internet] 2012; 126(3) [acesso em 17 jul 2015]. Disponível: http://dx.doi.org/10.1111/j.1600-0447.2012.01850.x.

27. Veras R. Envelhecimento populacional contemporâneo: demandas, desafios e inovações. Rev. Saúde Pública. [Internet] 2009; 43(3) [acesso em 20 mai 2015]. Disponível: http://dx.doi.org/10.1590/S0034-89102009005000025.

28. Ando NM, Targa LV, Almeida A, Silva DHS, de Barros EF, Schwalm FD, et al. Declaração de Brasília. O conceito de rural e o cuidado à saúde. Rev bras med fam comunidade. [Internet] 2011; 6(19) [acesso em 5 fev 2016]. Disponível: http://dx.doi.org/10.5712/rbmfc6(19)390. 